\title{
NOVOS REGISTROS DE APHYLLOPHORALES PARA O BRASIL ${ }^{1}$
}

\author{
Tatiana Baptista Gibertoni² \\ Maria Auxiliadora de Queiroz Cavalcanti ${ }^{2}$
}

Recebido em 07/02/2000. Aceito em 28/04/2000

\begin{abstract}
RESUMO - (Novos registros de Aphyllophorales para o Brasil). De coletas efetuadas em três remanescentes de Mata Atlântica de Pernambuco, no período de outubro/1997 a setembro/1998, foram identificadas, entre outras espécies de Aphyllophorales, duas espécies que estão sendo referidas pela primeira vez para o Brasil: Phellinus aureobruneus Wright \& Blumenfeld e Trichaptum abietinum (Dicks.: Fr) Ryv. Descrições macro e microscópicas, distribuição geográfica, discussão e ilustrações das espécies são fornecidas.
\end{abstract}

Palavras-chave - taxonomia, Aphyllophorales, Mata Atlântica, Pernambuco, Brasil

ABSTRACT - (New records of Aphyllophorales for Brazil). Aphyllophorales was collected between October/ 1997 and September/1998 in three Atlantic Rain Forest remnants in the State of Pernambuco, northeast Brazil. Two species are new records for Brazil: Phellinus aureobruneus Wright \& Blumenfeld and Trichaptum abietinum (Dicks.: Fr) Ryv. Macro and microscopic descriptions, geographyc distribution, discussion and species illustrations are provided.

Key words - taxonomy, Aphyllophorales, Atlantic Rain Forest, Pernambuco, Brazil

\section{Introdução}

A Mata Atlântica é uma formação vegetal litorânea caracterizada principalmente pela alta diversidade específica. Devido à sua localização, foi o primeiro alvo do impacto causado pela colonização e posterior urbanização do litoral, o que quase levou ao seu desaparecimento. Isso pode ser constatado em Pernambuco, onde os remanescentes da Mata Atlântica estão reduzidos a apenas $8,44 \%$ da cobertura original (Ministério do Meio Ambiente, dos Recursos
Hídricos e da Amazônia Legal 1998).

Apesar de inúmeros trabalhos envolverem os mais diversos aspectos da Mata Atlântica, escassos são os estudos sobre os fungos, observando-se algumas referências, especialmente aos pertencentes a Aphyllophorales no Nordeste do Brasil (Cavalcanti 1976; Lucena 1988; GóesNeto 1994). Este é um fato preocupante, pois este grupo de fungos é o decompositor primordial na maioria dos ecossistemas florestais e o estudo destes macromicetos nessas áreas é de vital importância para o conhecimento de sua

1 Parte da Dissertação de Mestrado da primeira autora

2 Departamento de Micologia, Universidade Federal de Pernambuco, Av. Prof. Nelson Chaves, s/n., CEP 50760-420, Recife, PE, Brasil 
biodiversidade. Além da degradação de madeira e de outros restos vegetais, animais, microbianos e até mesmo da micota, apresentando papel fundamental na reciclagem de nutrientes, alguns fungos dessa ordem são comestíveis (Pegler \& Spooner 1992), fitopatógenos (Stalpers \& Loerakker 1982), nematófagos (Tzean \& Liou 1993) e inibidores de térmitas (Grace et al. 1992).

O presente trabalho visa divulgar a primeira ocorrência de duas espécies de Aphyllophorales no Brasil, em áreas de Mata Atlântica do Estado de Pernambuco.

\section{Material e métodos}

Foram realizadas 12 coletas dos fungos Aphyllophorales em três áreas de remanescentes de Mata Atlântica de Pernambuco: Reserva Ecológica de Dois Irmãos, em área da Companhia Pernambucana de Saneamento (COMPESA), no município de Recife (8॰15’30'S e 35 $\left.57^{\prime} 00^{\prime \prime} W\right)$; Mata de Gurjaú, em área da COMPESA, no município de Cabo ( $8^{\circ} 14^{\prime} 12^{\prime \prime} \mathrm{S}$ e 3503'00"W); e Estação Ecológica de Tapacurá, câmpus avançado da Universidade Federal Rural de Pernambuco, no município de São Lourenço da Mata $\left(8^{\circ} 05^{\prime} \mathrm{S}\right.$ e $\left.35^{\circ} 13^{\prime} \mathrm{W}\right)$, pertencentes ao domínio da floresta ombrófila densa (Veloso et al. 1991). Os locais foram visitados de três em três meses, no período de outubro/1997 a setembro/1998.

No campo, os espécimes foram coletados manualmente com auxílio de faca e acondicionados em sacos de papel. No laboratório, foram feitas anotações relativas à cor das superfícies abhimenial e himenial, do contexto, dos tubos e da margem, utilizando-se a carta de cores de Maerz \& Paul (1950), e anotações relativas à largura, altura e espessura do basidiocarpo. $\mathrm{Na}$ preservação e herborização seguiu-se Fidalgo \& Bononi (1989), com modificações relativas à secagem do material, que era deixado, no mínimo, uma semana em estufa a $50^{\circ} \mathrm{C}$. As espécies foram depositadas no Herbário Padre Camilo
Torrend (URM), do Departamento de Micologia da Universidade Federal de Pernambuco.

Para a observação microscópica do material, foram feitos cortes à mão livre de cada basidiocarpo, com lâminas de aço inoxidável. Os cortes foram acondicionados em lâminas de vidro e corados com hidróxido de potássio $3 \%$ e floxina $1 \%$, segundo a técnica de Martin (1934). Paralelamente, os mesmos foram corados com azul de Amann, de acordo com Kotlaba \& Pouzar (1964), que, além de demonstrar a cianofilia dos basidiosporos e das hifas, impede a dissolução de material cristalino (Reeves \& Welden 1967). Também foi utilizado o reagente de Melzer, segundo Singer (1951), de modo a se observar a reação amilóide ou dextrinóide dos basidiosporos, hifas e outras microestruturas.

$\mathrm{Na}$ identificação, foram usados, entre outros, trabalhos específicos como os de Gilbertson \& Ryvarden (1987); Larsen \& Cobb-Poule (1990); Overholts (1967); Ryvarden \& Gilbertson (1993); Ryvarden (1976;1991); Teixeira (1993; 1994); Wright \& Blumenfeld (1984).

\section{Resultados e discussão}

Dentre as 42 espécies de Aphyllophorales coletadas em três áreas de remanescentes de Mata Atlântica de Pernambuco, duas destacaram-se por constituírem a primeira ocorrência para o Brasil, sendo uma espécie da família Hymenochaetaceae e uma da família Polyporaceae.

\section{Família Hymenochaetaceae}

1. Phellinus aureobruneus Wright \& Blumenfeld, Mycotaxon 21: 4171984.

(Fig. 1a e b)

Basidiocarpo anual, dimidiado, 7,0x4,5x0,5$3,0 \mathrm{~cm}$. Superfície abhimenial velutina, azonada, amarelada, MP13J12 (Titian). Margem obtusa, inteira, marrom alaranjado, MP13L11 (Peruvian Br.). Contexto 0,3-1,0cm espesso, amarelo dourado, MP9L7 (Cavalary Deep Chrome). Super- 


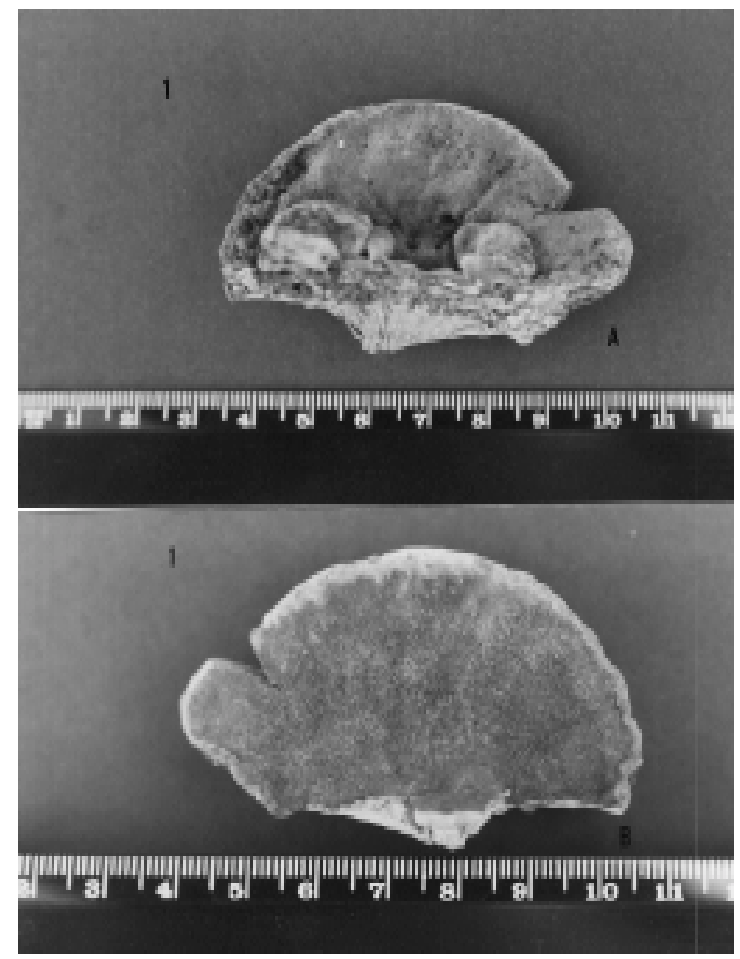

Figura 1. Phelinus aureobruneus. a) superfície abhimenial, b) superfície himenial.

fície himenial poróide, marrom escura, MP16L12, 9-8 poros $/ \mathrm{mm}$, circulares a angulares. Sistema hifálico dimítico; hifas generativas com septo simples, hialinas, parede fina, 2,5-2,7 $\mu \mathrm{m}$ diâm.; hifas esqueléteas marrons, não ramificadas, parede espessa, 4,5-5,0 $\mathrm{mm}$ diâm. Setas e cistídios ausentes. Basídios clavados a subglobosos, 7,5 $\mu \mathrm{m}$ diâm., tetraesporados. Basidiosporos marromdourados, globosos, parede fina, 2,7-4,5 $\mu$ m diâm.

Material examinado: BRASIL. Pernambuco: Recife, Reserva Ecológica de Dois Irmãos, em angiosperma indeterminada em decomposição, X/1997, Gibertoni, (URM 76718).

Distribuição geográfica: $P$. aureobruneus só é conhecido de sua localidade-tipo, a Argentina, sendo esta a primeira citação para o Brasil e o segundo relato da espécie.

Os espécimes apresentaram caracteres idênticos aos da descrição original de $P$. aureobruneus, diferindo apenas na ausência de sulcos na superfície abhimenial e da linha preta na base do contexto. Esta espécie difere de $P$. rheicolor (Lloyd) Ryv. e de P. rhabarbarnus (Berk.) Cunn. pela ausência de setas.

\section{Família Polyporaceae}

1. Trichaptum abietinum (Dicks.: Fr.) Ryv., Norw. J. Bot 19: 2371972.

(Fig. 2a e b)

Basiônimo: Polyporus abietinus Dicks.: Fr., Syst. Mycol. 1: 3701821

Basidiocarpo anual, pileado, usualmente imbricado, píleo rígido a coriáceo, 0,4-2,5 x 0,6$1,5 \mathrm{~cm}$. Superfície abhimenial tomentosa a velutina, zonada, parda, MP12B4 (Long Beach+), margem sinuosa, branca, MP25A1. Contexto reduzido, $0,1 \mathrm{~cm}$ espesso, marrom-claro, MP15A5 (Log Cabin+). Superfície himenial poróide, marrom escura, MP16A7 (Eagle/Clove Brown), 5 poros $/ \mathrm{mm}$, dissepimento denteado a lacerado. Sistema hifálico dimítico; hifas generativas hialinas, finas a levemente espessadas, com ansas, 2,5-3,7 $\mu \mathrm{m}$ diâm.; hifas esqueléteas hialinas, com parede espessada, 2,54,5 $\mu \mathrm{m}$ diâm. Cistídios numerosos, clavados, lisos ou encrustados no ápice, 11,8-13,7 x 3,6$4,5 \mu \mathrm{m}$. Basidiosporos cilíndricos a alantóides, li-

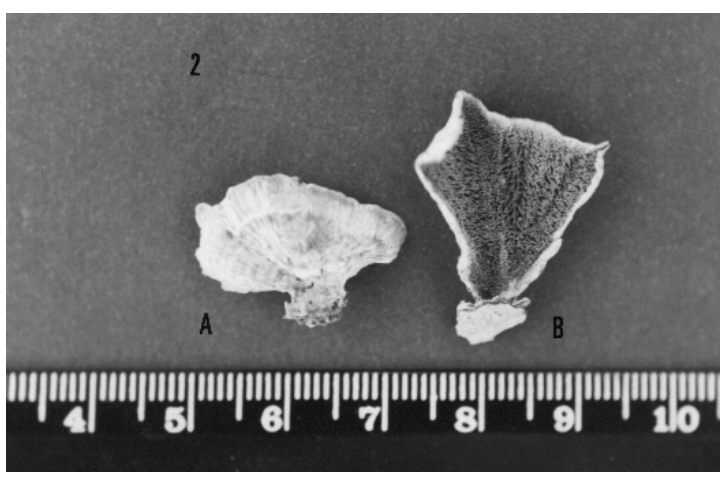

Figura 2. Trichaptum abietinum. a) superfície abhimenial, b) superfície himenial.

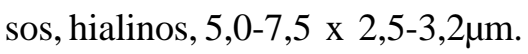

Material examinado: BRASIL. Pernambuco: Recife, Reserva Ecológica de Dois Irmãos, em angiosperma indeterminada em decomposição, X/1997, Gibertoni, (URM 76764); 
IV/1998, Gibertoni, (URM 76765).

Distribuição geográfica: Venezuela (Fidalgo 1968, como Hirchioporus abietinus (Dicks.: Fr.) Donk); comum na Europa, Ásia e América do Norte (Ryvarden 1976); restrito à Zona Temperada do Norte (Gilbertson \& Ryvarden 1987; Ryvarden \& Gilbertson 1993); Croácia (Tortic 1992); Sibéria central (Zhukoff 1995); China (Zhang 1997); Grécia (Zervakis et al. 1998).

Trata-se da primeira citação para o Brasil.

Gilbertson \& Ryvarden (1987) consideram a espécie como pioneira em troncos recém-caídos, além de poder variar de completamente ressupinada a pileada. O conceito de $T$. abietinum de Overholts (1967) incluía T. fuscoviolaceum e $T$. laricinum, mas testes de interfertilidade (Macrae 1967) demonstraram que as três espécies são diferentes.

\section{Referências bibliográficas}

Cavalcanti, M. A. Q. 1976. Introdução ao conhecimento dos basidiomicetos poliporóides da Zona da Mata de Pernambuco. Tese de Livre-docência, Universidade Federal de Pernambuco, Recife.

Fidalgo, O. 1968. A preliminary enumeration of Venezuelan Polyporaceae. Acta Biológica Venezuelana 6(2): 1-37.

Fidalgo, O. \& Bononi, V. L. R. (coords.). 1989. Técnicas de coleta, preservação e herborização de material botânico. Instituto de Botânica, São Paulo.

Gilbertson, R. L. \& Ryvarden, L. 1987. North American polypores. Vol. 2. Fungiflora, Oslo.

Góes-Neto, A. 1994. Diagnóstico da biodiversidade de macromicetos do Estado da Bahia: evolução histórica e situação atual. Monografia de Bacharelado. Universidade Federal da Bahia, Salvador.

Grace, J. K.; Goodwell, B. S.; Jones, W .E .; Chandhoke, V. \& Jellison, J. 1992. Evidence for inhibition of termite (Isoptera: Rhinotermitidade) feeding by extracellular metabolites of a wood decay fungus. Proceedings, Haiwaiian Entomological Society 31: 249-252.

Kotlaba, F. \& Pouzar, Z. 1964. Preliminary results on the staining of the spores and other structures of Homobasidiomycetes in cotton blue and its importance for taxonomy. Transactions of the British Mycological Society 47: 653-654.

Larsen, M. \& Cobb-Poule, L. A. 1990. Phellinus (Hymenochaetaceae). A survey of the world taxa.
Synopsis Fungorum 3, Fungiflora, Oslo.

Lucena, V. L. A. 1988. Estudo da família Ganodermataceae Donk na Mata do Buraquinho, João Pessoa, Paraíba, Brasil. Dissertação de Mestrado. Universidade Federal de Pernambuco, Recife.

Macrae, R. 1967. Pairing incompatibility and other distinctions among Hirschioporus (Polyporus) abietinus, $H$. fusco-violaceus, and $H$. laricinus. Canadian Journal of Botany 45: 1371-1383.

Maerz, A. \& Paul, M. R. 1950. A dictyonary of colour. McGraw-Hill Book Company, $2^{\text {a }}$ ed., New York.

Martin, C. G. 1934. Three new Heterobasidiomycetes. Mycologia 26: 261-265.

Ministério do Meio Ambiente, dos Recursos Hídricos e da Amazônia Legal. 1998. Primeiro relatório nacional para a convenção sobre diversidade biológica - Brasil. Brasília.

Overholts, L. O. 1967. The Polyporaceae of the United States, Alaska and Canada. Ann Arbor: University of Michigan.

Pegler, D. N. \& Spooner, B. 1992. The mushroom identifier. The Apple Press, London.

Reeves, F. \& Welden, A. L. 1967. West Indian species of Hymenochaete. Mycologia 59: 1034-1049.

Ryvarden, L. 1976. The Polyporaceae of North Europe. Vol. 2. Fungiflora, Oslo.

Ryvarden, L. 1991. Genera of Polypores Nomenclature and Taxonomy. Synopsis Fungorum 5, Fungiflora, Oslo.

Ryvarden, L. \& Gilbertson, R. L. 1993. European Polypores. Part 2. Synopsis Fungorum 6, Fungiflora, Oslo.

Singer, R. 1951. The Agaricales (mushrooms) in modern taxonomy. Lilloa, Tocumán.

Stalpers, J. A. \& Loerakker, W. M. 1982. Laetisaria and Limonomyces species (Corticiaceae) causing pink diseases in turf grasses. Canadian Journal of Botany 60: 529-537.

Teixeira, A. R. 1993. Chave para identificação dos gêneros de Polyporaceae com base na morfologia do basidiocarpo. Boletim do Instituto de Botânica $\mathbf{n}^{\circ} 8$.

Teixeira, A. R. 1994. Genera of Polyporaceae: an objective approach. Boletim da Chácara Botânica de Itu n $\mathbf{n}^{\circ} 1$.

Tortic, M. 1992. Macromycetes of the Gorski Kotar (Croatia) III. Acta Botanica Croatica 51: 113-130.

Tzean, S. S. \& Liou, J. Y. 1993. Nematophagous resupinate basidiomycetous fungi. Phytopathology 83(10): 1015-1020. 
Veloso, H. P., Rangel Filho, A. L. R. \& Lima, J. C. A. 1991. Classificação da vegetação brasileira, adaptada a um sistema universal. Fundação Instituto Brasileiro de Geografia e Estatística, Rio de Janeiro.

Zervakis, G., Dimou, D. \& Balis, C. 1998. A check-list of the greek macrofungi including hosts and biogeografic distribution: I. Basidiomycotina. Mycotaxon 66: 273-336.
Zhang, X. 1997. Fungal flora of Daba Mountains: Aphyllophorales and some Basidiomycota. Mycotaxon 61: 41-45.

Zhukoff, E. A. 1995. Aphyllophorales (Basidiomycetes) from central Siberia. Mycotaxon 53: 437 445.

Wright, J. E. \& Blumenfeld, S. N. 1984. New south american species of Phellinus (Hymenochaetaceae). Mycotaxon 21: 413-425. 